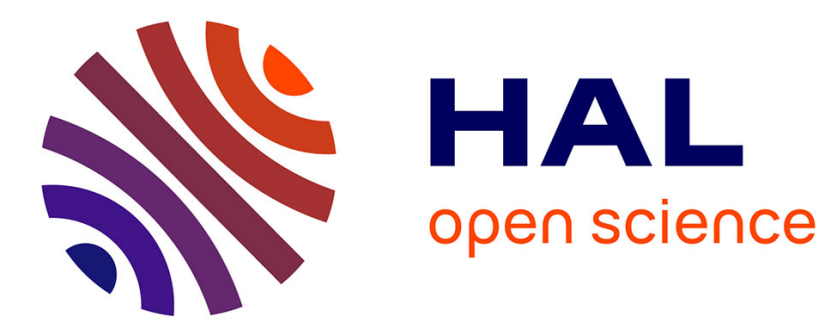

\title{
Two imperfect repair models for a gamma deteriorating system: A comparison
}

Sophie Mercier, I T Castro

\section{To cite this version:}

Sophie Mercier, I T Castro. Two imperfect repair models for a gamma deteriorating system: A comparison. ESREL 2018 (Annual European Safety and Reliability Conference), Jun 2018, Trondheim, Norway. hal-01812171

\section{HAL Id: hal-01812171 \\ https://hal.science/hal-01812171}

Submitted on 11 Jun 2018

HAL is a multi-disciplinary open access archive for the deposit and dissemination of scientific research documents, whether they are published or not. The documents may come from teaching and research institutions in France or abroad, or from public or private research centers.
L'archive ouverte pluridisciplinaire HAL, est destinée au dépôt et à la diffusion de documents scientifiques de niveau recherche, publiés ou non, émanant des établissements d'enseignement et de recherche français ou étrangers, des laboratoires publics ou privés. 


\title{
Two imperfect repair models for a gamma deteriorating system: A comparison
}

\author{
Sophie Mercier \\ Univ Pau \& Pays Adour / CNRS, IPRA-LMAP, 64000 PAU, France \\ sophie.mercier@univ-pau.fr \\ I.T. Castro \\ Department of Mathematics, University of Extremadura, Spain \\ inmatorres@unex.es
}

\begin{abstract}
A system is considered, which is deteriorating over time according to a non-homogeneous gamma process. The point of the presentation is to propose and compare two models of imperfect repairs for the system. For sake of simplicity, only periodic (and instantaneous) repairs are here envisioned. The first model, called the Arithmetic Reduction of Deterioration of order 1 (ARD1), assumes that a repair removes a given proportion of the degradation accumulated by the system from the last maintenance action. The second model, called Arithmetic Reduction of Age of order 1 (ARA1), refers to the virtual age models proposed by Kijima (1989) and further studied by Doyen \& Gaudoin (2004) in the context of recurrent events: the ARA1 model assumes that a repair reduces the age accumulated by the system since the last maintenance action, in a given proportion. An ARD1 repair hence lowers the deterioration level, without rejuvenating the system. On the contrary, by an ARA1 repair, the system is put back to the exact situation where it was some time before, which entails the lowering of both its deterioration level and (virtual) age. The two models may hence correspond to different maintenance actions in an applicative context. This presentation focuses on the comparison between the two models, from a probabilistic point of view (moments and stochastic ordering). An application in a maintenance optimization context is also provided, for illustration purpose. A specific case is analyzed, where the two repair models provide identical expected deterioration levels at maintenance times ("equivalent" case). The comparison results can help understanding which among the two models is the best adapted in an applicative context.
\end{abstract}

\section{INTRODUCTION}

Many systems suffer a physical degradation before they fail. This degradation is a complex process as it depends of many factors (material, stress loads, temperature, ...). To mitigate the effect of the system degradation and to extend the system lifetime, a large volume of maintenance models have been proposed in the literature, with different maintenance actions. Most of these models are limited to perfect repairs (Huynh et al. 2014, Caballé et al. 2015, Hong et al. 2014 among others). However, imperfect maintenance actions describe more realistic situations than perfect repairs. Some advances have been made to include imperfect repairs in a degrading system. Alaswad \& Xiang 2017 classified the impact of the maintenance actions over the maintained system into three types. The first type assumes that the maintenance actions return the system to a previous stage of deterioration. In the second type, the imperfect maintenance reduces the degradation level of the maintained system (Castro \& Mercier 2016). The third approach is based on the idea that the maintenance action changes the rate of degradation of the system (Zhang et al. 2015). However, as Zhang et al. 2015 claimed, the issue of treating imperfect maintenance in the context of degrading systems remains widely open nowadays.

Following the spirit showed in (Mercier \& Castro 2013) and (Castro \& Mercier 2016), two models of imperfect repair are here proposed and compared for a system accumulating deterioration over time. The first model, called the Arithmetic Reduction of Deterioration of order 1 (ARD1), assumes that the repair removes the proportion $\rho$ of the degradation accumulated by the system from the last maintenance action (with $\rho \in(0,1)$ ). The second model, called Arithmetic Reduction of Age of order 1 (ARA1), refers 


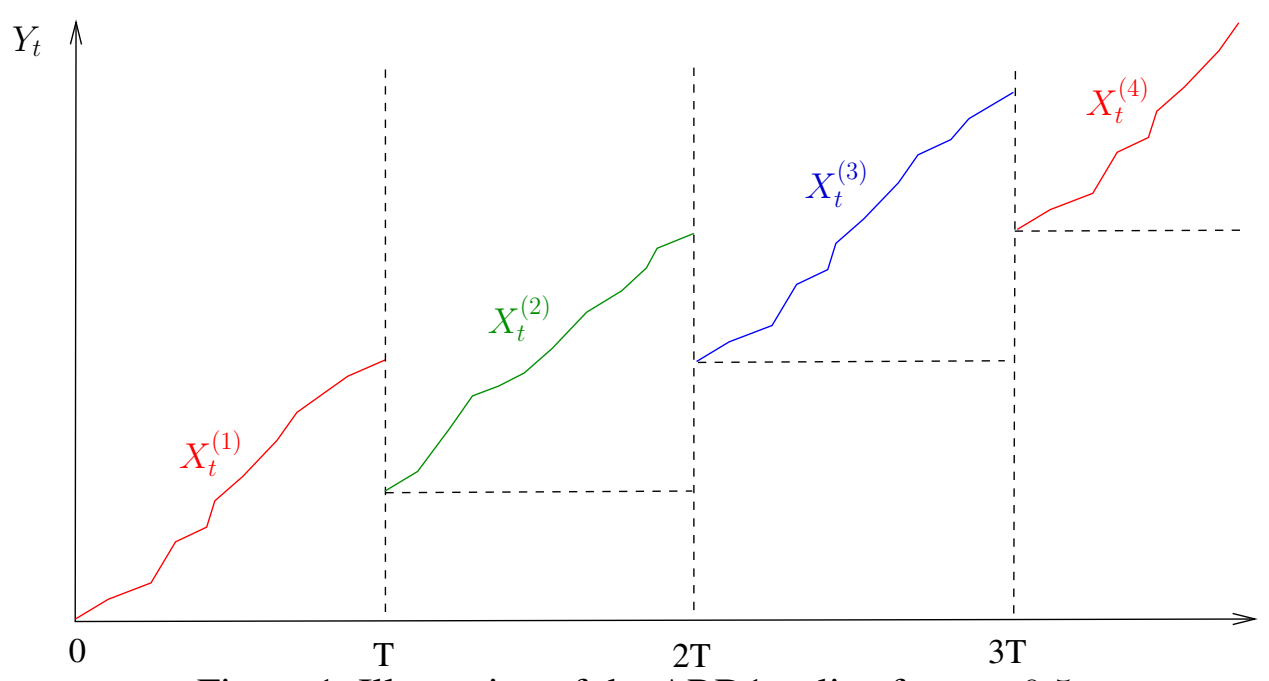

Figure 1: Illustration of the ARD1 policy for $\rho=0.5$

to the virtual age models proposed by (Kijima 1989) and further studied by (Doyen \& Gaudoin 2004) in the context of recurrent events: the ARA1 model assumes that the repair removes the proportion $\rho$ of the age accumulated by the system since the last maintenance action. An ARD1 repair hence lowers the deterioration level, without rejuvenating the system. On the contrary, by an ARA1 repair, the system is put back to the exact situation where it was some time before, which entails the lowering of both its deterioration level and (virtual) age. The two models may hence correspond to different maintenance actions in an applicative context.

For a better understanding of the differences between the two models, this paper focuses on their comparison, from a probabilistic point of view. Assuming that the degradation of the system is modeled by a non homogeneous gamma process, stochastic comparisons of both location and spread of the two resulting processes are given. Moreover, a specific case is analyzed, where the two models provide identical expected deterioration levels at repair times ("equivalent" case). Finally, an illustration of the two models is provided, by including them in a global maintenance policy, with replacement of the system when it is too deteriorated.

The paper is organized as follows: The two models of imperfect repair are described in Section 2. The comparison results are given in Section 3 (including the "equivalent" case). Section 4 deals with the application to the global maintenance strategy and concluding remarks are provided in Section 5, together with possible extensions.

\section{THE TWO MODELS OF IMPERFECT REPAIRS}

\subsection{The intrinsic deterioration and notations}

For $a, b>0$, let us first recall that the gamma distribution $\Gamma(a, b)$ with parameters $(a, b)$ admits the follow- ing p.d.f. (probability distribution function):

$f_{a, b}(x)=\frac{b^{a}}{\Gamma(a)} x^{a-1} e^{-b x}, \forall x>0$.

The corresponding mean and variance are $\frac{a}{b}$ and $\frac{a}{b^{2}}$, respectively.

A system is considered with degradation modeled by a non homogeneous gamma process $\left(X_{t}\right)_{t>0}$ with parameters $A(\cdot)$ and $b$, where $A(\cdot): \mathbb{R}_{+} \longrightarrow \mathbb{R}_{+}$is continuous and non-decreasing with $A(0)=0$, and $b>0$. We recall that $\left(X_{t}\right)_{t>0}$ is a process with independent increments such that $X_{0}=0$ almost surely and such that each increment $X_{t+s}-X_{t}$ is gamma distributed $\Gamma(A(t+s)-A(t), b)$ for all $s, t>0$.

The system is periodically and instantaneously repaired each $T$ units of time. For modeling purpose, we set $X^{(i)}, i \in \mathbb{N}^{*}$ to be i.i.d. copies of $X=\left(X_{t}\right)_{t \geq 0}$, where $X^{(i)}$ describes the evolution of the deterioration level between the $i$-th and $(i+1)$-th maintenance actions. For each imperfect repair model, the maintenance efficiency is measured by an Euclidian parameter $\rho \in(0,1)$.

\subsection{First model: Arithmetic Reduction of Deterioration of order 1 (ARDI)}

In this model, the maintenance action instantaneously removes the proportion $\rho$ of the degradation accumulated by the system from the last maintenance action (or from the origin). We set $\left(Y_{t}\right)_{t>0}$ be the process that describes the degradation level of the maintained system under this model of repair.

The ARD1 model is sketched in Figure 1 for $\rho=$ 0.5. It is developed as follows: At the beginning, the system deteriorates according to $X^{(1)}$. It is first maintained at time $T$, where the proportion $\rho$ of the accumulated deterioration since the origin is removed. This provides:

$$
Y_{t}=X_{t}^{(1)} \quad t<T, \quad Y_{T}=(1-\rho) X_{T}^{(1)} .
$$




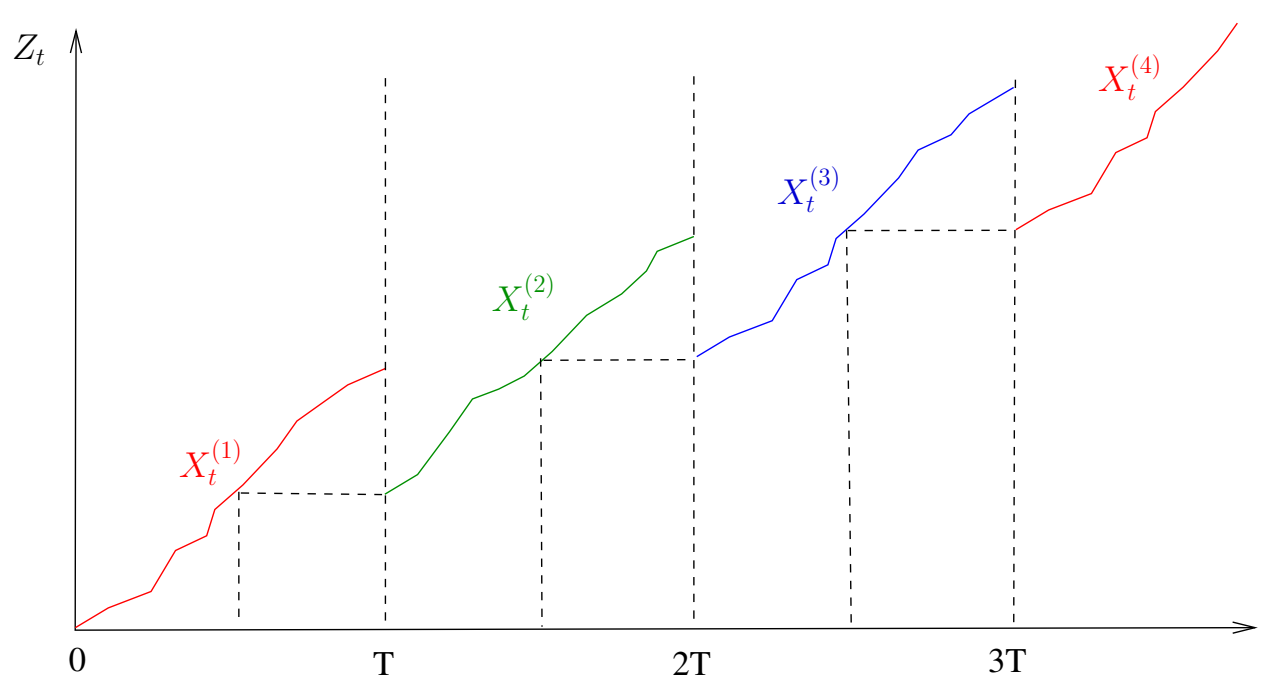

Figure 2: Illustration of the ARA1 policy for $\rho=0.5$

Between $T$ and $2 T$, the system deteriorates according to $X^{(2)}$. The age of the system is unchanged at time $T$ and we have

$Y_{t}=Y_{T}+\left(X_{t}^{(2)}-X_{T}^{(2)}\right)$

for all $T \leq t<2 T$. At the second maintenance time $2 T$, the proportion $\rho$ of the degradation accumulated between $T$ and $2 T$ is removed, which provides:

$Y_{2 T}=Y_{T}+(1-\rho)\left(X_{2 T}^{(2)}-X_{T}^{(2)}\right)$.

More generally, we have:

$Y_{t}=Y_{n T}+\left(X_{t}^{(n+1)}-X_{n T}^{(n+1)}\right)$

for all $n T \leq t<(n+1) T$, where $X_{t}^{(n+1)}-X_{n T}^{(n+1)}$ is gamma distributed $\Gamma(A(t)-A(n T), b)$ and

$Y_{(n+1) T}=Y_{n T}+(1-\rho)\left(X_{(n+1) T}^{(n+1)}-X_{n T}^{(n+1)}\right)$.

This provides

$Y_{n T}=(1-\rho) \sum_{i=1}^{n}\left(X_{i T}^{(i)}-X_{(i-1) T}^{(i)}\right)$

with $\Gamma\left(A(n T), \frac{b}{1-\rho}\right)$ as distribution.

When $\rho \rightarrow 1^{-}$, then $Y_{n T} \rightarrow 0^{+}$and the system is renewed at time $n T$ (As Good As New repair: AGAN). When $\rho \rightarrow 0^{+}$, the repair is ineffective and it is As Bad As Old (ABAO).

Except for the case $\rho \rightarrow 0^{+}$, if $t \bmod T \neq 0, Y_{t}$ is the sum of two independent and gamma distributed random variables (r.v.s) with different scale parameters, and it is not gamma distributed. Its expectation and variance are given by:

$$
\begin{aligned}
\mathbb{E}\left(Y_{t}\right) & =\frac{A(t)-\rho A(n T)}{b}, \\
\operatorname{var}\left(Y_{t}\right) & =\frac{A(t)-\rho(2-\rho) A(n T)}{b^{2}},
\end{aligned}
$$

for $n T \leq t<(n+1) T$.

It is easy to check that $\mathbb{E}\left(Y_{t}\right)$ and $\operatorname{var}\left(Y_{t}\right)$ are decreasing with respect to $\rho$, that is, the more efficient the repair, the smaller the expectation and variance of the deterioration level. In this way, the expectation and variance are minimal when $\rho \rightarrow 1^{-}$that is when the repair is AGAN, and maximal when $\rho \rightarrow 0^{+}$that is when the repair is ABAO.

\subsection{Second model: Arithmetic Reduction of (virtual) Age of order 1 (ARAl)}

As told in the introduction, the ARA1 model is based on the notion of virtual age, which is reduced by each maintenance action: each repair removes the proportion $\rho$ of the age accumulated by the system since the last maintenance action (or from the origin). This means that, at each maintenance action, the system goes back into its past: the deterioration level is hence reduced (just as for the ARD1 model) but the system is also rejuvenated at the same time (it becomes younger). In case of an increasing deterioration rate $(A(\cdot)$ convex), the rate of deterioration is hence reduced by the repair together with the deterioration level. We set $\left(Z_{t}\right)_{t>0}$ be the process that describes the degradation level of the maintained system under this model of repair.

The ARA1 model is sketched in Figure ?? for $\rho=0.5$. It is developed as follows: At the first maintenance time $T$, the (virtual) age of the system is suddenly reduced of $\rho T$, so that it becomes $T-\rho T=$ $(1-\rho) T$. This provides:

$Z_{t}=X_{T}^{(1)}$ for $t<T, \quad Z_{T}=X_{(1-\rho) T}^{(1)}$.

For $T \leq t<2 T$, the age of the system is $(1-\rho) T+$ $(t-T)=t-\rho T$. We get:

$Z_{t}=Z_{T}+\left(X_{t-\rho T}^{(2)}-X_{(1-\rho) T}^{(2)}\right)$.

At time $2 T^{-}$(just before the repair), the age of the system is $2 T-\rho T$ which is reduced of $\rho T$ at time $2 T$. 
The age hence is $2(1-\rho) T$ at time $2 T$. This provides:

$Z_{2 T}=Z_{T}+\left(X_{2(1-\rho) T}^{(2)}-X_{(1-\rho) T}^{(2)}\right)$.

More generally, for $n T \leq t<(n+1) T$, the virtual age of the system at time $t$ is $t-\rho n T$ (which is just the same as for an ARA1 model for recurrent events, see (Doyen \& Gaudoin 2004)). We obtain:

$Z_{t}=Z_{n T}+\left(X_{t-\rho n T}^{(n+1)}-X_{(1-\rho) n T}^{(n+1)}\right)$,

for all $n T \leq t<(n+1) T$, where $X_{t-\rho n T}^{(n+1)}-X_{(1-\rho) n T}^{(n+1)}$ is gamma distributed $\Gamma(A(t-\rho n T)-A((1-\rho) n T), b)$ and

$Z_{(n+1) T}=Z_{n T}+\left(X_{(1-\rho)(n+1) T}^{(n+1)}-X_{(1-\rho) n T}^{(n+1)}\right)$.

Hence

$Z_{n T}=\sum_{i=1}^{n}\left(X_{(1-\rho) i T}^{(i)}-X_{(1-\rho)(i-1) T}^{(i)}\right)$

and it is gamma distributed $\Gamma(A((1-\rho) n T), b)$. Here, $Z_{t}$ is the sum of two independent gamma distributed r.v.s which share the same scale parameter $b$ and it is gamma distributed $\Gamma(A(t-\rho n T), b)$. Also:

$\mathbb{E}\left(Z_{t}\right)=\frac{A(t-\rho n T)}{b}, \quad \operatorname{var}\left(Z_{t}\right)=\frac{A(t-\rho n T)}{b^{2}}$

for all $n T \leq t<(n+1) T$.

Here again, it is easy to check that $\mathbb{E}\left(Z_{t}\right)$ and $\operatorname{var}\left(Z_{t}\right)$ are decreasing with respect to $\rho$. Also, the cases $\rho \rightarrow 1^{-}$and $\rho \rightarrow 0^{+}$correspond to AGAN and $\mathrm{ABAO}$ repairs, respectively.

\section{COMPARISON RESULTS}

\subsection{Comparison of the moments}

We now come to the main object of the paper, which is the comparison between the two models of imperfect repairs. Note that, in an applicative context, there is no reason why the estimated repair efficiency should be the same when the impact of the maintenance is modeled by an ARD1 or ARA1 model. Considering two different efficiency parameters $\rho_{i}, i=1,2$, our point here is to compare $Y_{t}^{(1)}$ and $Z_{t}^{(2)}$, where exponent ${ }^{(i)}$ refers to $\rho_{i}$ for $i=1,2$. We begin with the comparison of their respective means and variances.

Due to the reduced size of the paper, results on the comparison of moments are provided only in case of a power-law shape function for the gamma process. The interested reader will find general results in an extended version of the paper (Mercier and Castro, submitted), as well as proofs for all the results of the paper.
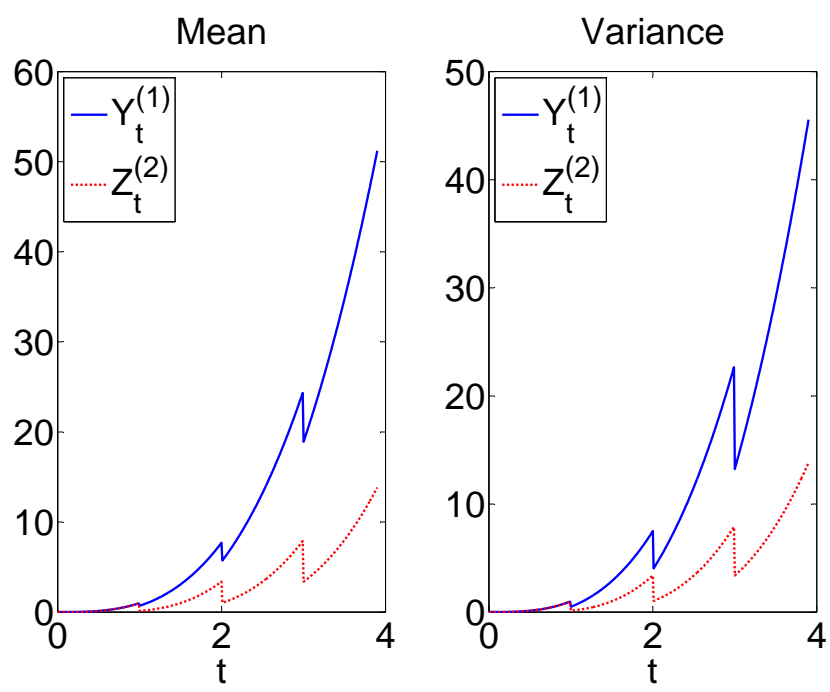

Figure 3: Mean and variance of $Y_{t}^{(1)}$ and $Z_{t}^{(2)}$ with respect to $t$ for $A(t)=t^{3}, b=1, \rho_{1}=0.95, \rho_{2}=0.5$, $T=1$

Proposition 1 Let $A(t)=\alpha t^{\beta}$ with $\alpha, \beta>0$. Assume $\beta \leq 1$.

1. We have

$$
\mathbb{E}\left(Z_{t}^{(2)}\right) \geq \mathbb{E}\left(Y_{t}^{(1)}\right), \forall t>0
$$

if and only if $\left(1-\rho_{2}\right)^{\beta} \geq\left(1-\rho_{1}\right)$.

2. We have

$$
\operatorname{Var}\left(Z_{t}^{(2)}\right) \geq \operatorname{Var}\left(Y_{t}^{(1)}\right), \forall t>0
$$

if and only if $\left(1-\rho_{2}\right)^{\beta} \geq\left(1-\rho_{1}\right)^{2}$.

If $\beta \geq 1$, all results are valid with reversed inequalities.

For illustration purpose, we consider $A(t)=t^{3}$, $b=1, T=1$ and $\rho_{2}=0.5$. As a first case, we take $\rho_{1}=$ 0.3 so that the conditions of the previous proposition are fulfilled for both expectation and variance. As expected, Figure 3 shows that $\mathbb{E}\left(Y_{t}^{(1)}\right)$ and $\mathbb{E}\left(Z_{t}^{(2)}\right)$ are ordered in the same way on the whole real line, the same for the variance. As a second case, we take $\rho_{1}=0.95$ so that the conditions are not fulfilled neither for expectation and variance. Figure 4 shows that $\mathbb{E}\left(Y_{t}^{(1)}\right)$ and $\mathbb{E}\left(Z_{t}^{(2)}\right)$ are not ordered in the same way on the whole real line, the same for the variance.

\subsection{Technical reminders}

Before providing stochastic comparison results between $Y_{t}^{(1)}$ and $Z_{t}^{(2)}$, we here recall a few definitions and results from the literature.

As a first step, let us recall that given two non negative random variables $X$ and $Y$ with probability distribution functions $f_{X}$ and $f_{Y}$, and survival functions $\bar{F}_{X}$ and $\bar{F}_{Y}$, respectively, then: 

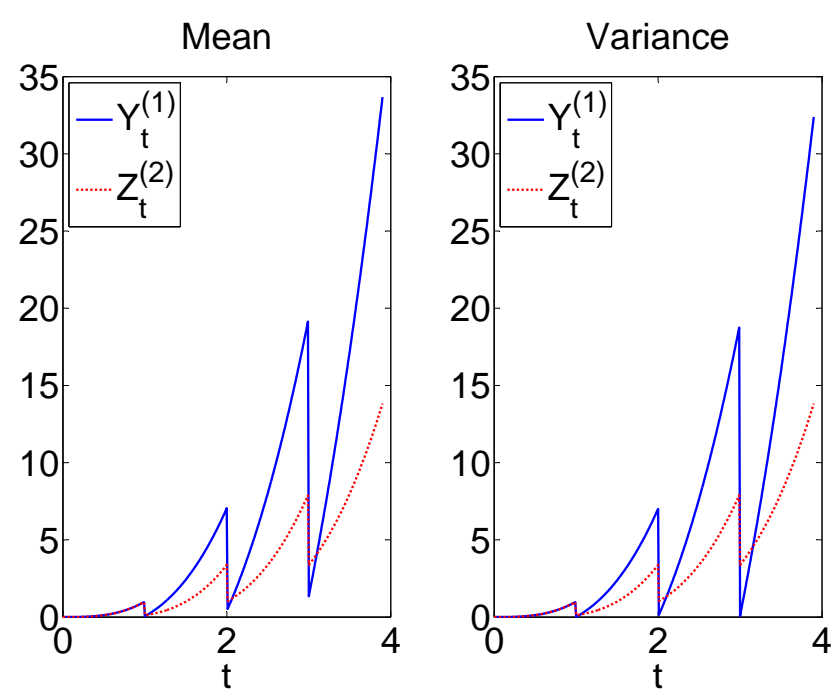

Figure 4: Mean and variance of $Y_{t}^{(1)}$ and $Z_{t}^{(2)}$ with respect to $t$ for $A(t)=t^{3}, b=1, \rho_{1}=0.95, \rho_{2}=0.5$, $T=1$

1. $X$ is said to be smaller than $Y$ in the usual stochastic order $\left(X \prec_{\text {sto }} Y\right)$ if $\bar{F}_{X} \leq \bar{F}_{Y}$;

2. $X$ is said to be smaller than $Y$ in the likelihood ratio order $\left(X \prec_{l r} Y\right)$ if $\frac{f_{Y}}{f_{X}}$ is non-decreasing;

3. $X$ is said to be smaller than $Y$ in the convex (concave) order $\left(X \prec_{c x(c v)} Y\right)$ if $\mathbb{E}(\varphi(X)) \leq$ $\mathbb{E}(\varphi(Y))$ for all convex functions $\varphi$ (provided the expectations exist);

4. $X$ is said to be smaller than $Y$ in the increasing convex (concave) order $\left(X \prec_{i c x(i c v)} Y\right)$ if $\mathbb{E}(\varphi(X)) \leq \mathbb{E}(\varphi(Y))$ for all increasing convex (concave) functions $\varphi$ (provided the expectations exist).

The usual stochastic order and the likelihood ratio order compare the locations of random variables whereas increasing convex (concave) orders also compare their variability: $X \prec_{i c x}\left(\prec_{i c v}\right) Y$ roughly means that $\mathbb{E}(X) \leq \mathbb{E}(Y)$ (location condition) plus the fact that $X$ is less (more) "variable" than $Y$, in a stochastic sense. Also, $X \prec_{c x}\left(\prec_{c v}\right) Y$ is equivalent to $X \prec_{i c x}\left(\prec_{i c v}\right) Y$ plus $\mathbb{E}(X)=\mathbb{E}(Y)$.

The likelihood ratio order is known to imply the usual stochastic order, which itself implies both increasing convex and concave orders, see Müller \& Stoyan 2002 or Shaked \& Shanthikumar 2007 for more details.

We finally review known results on the comparison of gamma distributions in the following lemma, see (Müller \& Stoyan 2002, p. 62) for instance.

Lemma 1 Let $X$ and $Y$ be gamma distributed random variables with parameters $\left(a_{1}, b_{1}\right)$ and $\left(a_{2}, b_{2}\right)$, respectively, where $a_{i}, b_{i}>0$ for $i=1,2$. Then:

1. If $a_{1} \leq a_{2}$ and $b_{1} \geq b_{2}$, then $X \prec_{l r} Y$;
2. If $a_{1} \geq a_{2}$ and $a_{1} / b_{1} \leq a_{2} / b_{2}$, then $X \prec_{i c x} Y$;

3. If $a_{1} \leq a_{2}, b_{1} \leq b_{2}$ and $a_{1} / b_{1} \leq a_{2} / b_{2}$, then $X \prec_{i c v} Y$.

The previous lemma is the basis for deriving the stochastic comparison results between the different imperfect repair models given in the next subsection.

\subsection{Stochastic comparison results}

As a first step, the influence of the efficiency parameter $\rho$ on the deterioration level is studied for each imperfect repair model.

\section{Proposition 2 We have:}

1. $Y_{n T}$ decreases with respect to $\rho$ in the sense of the likelihood order: If $\rho_{1}<\rho_{2}$, then $Y_{n T}^{(2)} \prec_{l r} Y_{n T}^{(1)}$;

2. $Y_{t}$ decreases with respect to $\rho$ in the sense of both increasing convex and concave orders: If $\rho_{1}<$ $\rho_{2}$, then $Y_{t}^{(2)} \prec_{i c x} Y_{t}^{(1)}$ and $Y_{t}^{(2)} \prec_{i c v} Y_{t}^{(1)}$;

3. $Z_{t}$ decreases with respect to $\rho$ for the likelihood ratio order (and hence also for both increasing convex and concave orders): If $\rho_{1}<\rho_{2}$, then $Z_{t}^{(2)} \prec_{l r} Z_{t}^{(1)}$.

In each case, we can see that as expected, the more efficient the maintenance action is (namely the larger $\rho$ is), the smaller the deterioration level is. Note however that, based on the fact that the likelihood ratio order is stronger than both increasing convex and concave orders, the results for the ARA1 model are stronger than for the ARD1 one. (Counter-examples can be found, which show that that $Y_{t}^{(1)}$ and $Y_{t}^{(2)}$ are not comparable for the likelihood ratio order in a general setting).

We now come to the stochastic comparison between the two models.

Theorem 1 If $A(\cdot)$ is concave and

$A\left(\left(1-\rho_{2}\right) t\right) \geq\left(1-\rho_{1}\right) A(t)$ for all $t$

(which is true if $\rho_{1} \geq \rho_{2}$ ), then $Y_{t}^{(1)} \prec_{i c x} Z_{t}^{(2)}$ for all $t \geq 0$.

If $A(t)$ is convex with a reversed inequality in (4), then $Z_{t}^{(2)} \prec_{i c v} Y_{t}^{(1)}$ for all $t \geq 0$.

As a specific case, if $\rho_{1}=\rho_{2}=\rho$, then $Y_{t} \prec_{i c x} Z_{t}$ for all $t \geq 0$ if $A(\cdot)$ is concave, and $Z_{t} \prec_{i c v} Y_{t}$ for all $t \geq 0$ if $A(\cdot)$ is convex. The concavity/convexity of the shape function $A(\cdot)$ of the underlying gamma process hence deeply infers on the comparison results between the two models: When $A(\cdot)$ is convex, the rejuvenation included in a ARA1 model leads to a lower deterioration level than for an ARD1 model.

Specific results are now summarized in the classical case of a homogeneous gamma process for both moments and stochastic comparisons. 
Corollary 1 Assume that $A(t)=\alpha t$ for all $t \geq 0$, where $\alpha>0$. We have the following results:

1. If $\rho_{1} \geq \rho_{2}$, then $Y_{t}^{(1)} \prec_{i c x} Z_{t}^{(2)}$ and hence $\mathbb{E}\left(Y_{t}^{(1)}\right) \leq \mathbb{E}\left(Z_{t}^{(2)}\right)$ for all $t \geq 0$;

2. If $\rho_{1} \leq \rho_{2}$, then $Z_{t}^{(2)} \prec_{i c v} Y_{t}^{(1)}$ and hence $\mathbb{E}\left(Z_{t}^{(2)}\right) \leq \mathbb{E}\left(Y_{t}^{(1)}\right)$ for all $t \geq 0 ;$

3. If $\rho_{1}=\rho_{2}$, then $Y_{t}^{(1)} \prec_{c x} Z_{t}^{(2)}$ and $Z_{t}^{(2)} \prec_{c v} Y_{t}^{(1)}$ and hence $\mathbb{E}\left(Z_{t}^{(2)}\right)=\mathbb{E}\left(Y_{t}^{(1)}\right)$ for all $t \geq 0$;

4. $\operatorname{Var}\left(Z_{t}^{(2)}\right) \geq \operatorname{Var}\left(Y_{t}^{(1)}\right)$ for all $t>0$ if and only if $1-\rho_{2} \geq\left(1-\rho_{1}\right)^{2}$.

\subsection{Mostly equivalent imperfect repair models}

In an applied context, parameters $\rho_{1}$ and $\rho_{2}$ for ARD1 and ARA1 models, respectively, will be estimated from feedback data, which will be typically gathered at maintenance times $i T, i \geq 1$. As a consequence, we can expect that the estimated parameters $\hat{\rho}_{1}$ and $\hat{\rho}_{2}$ should be such that the corresponding expected deterioration levels should be very similar at maintenance times, namely such that $\mathbb{E}\left(Y_{i T}^{(1)}\right) \simeq \mathbb{E}\left(Z_{i T}^{(2)}\right)$ for all $i \in \mathbb{N}^{*}$. Equivalently, the estimated parameters should be such that

$$
\frac{\left(1-\hat{\rho}_{1}\right) A(i T)}{b} \simeq \frac{A\left(\left(1-\hat{\rho}_{2}\right) i T\right)}{b} \text {, for all } i \geq 1 .
$$

There hence is a specific interest for the applications to compare the ARD1 and ARA1 models under the condition

$$
\left(1-\rho_{1}\right) A(i T)=A\left(\left(1-\rho_{2}\right) i T\right) \text { for all } i \geq 1,
$$

which will lead to equivalent deterioration levels at maintenance times. However, the previous requirement (5) does not seem to have a solution for a general shape function $A(\cdot)$. We hence restrict the study to the power-law case $A(t)=\alpha t^{\beta}$ (with $\alpha, \beta>0$ ), for which (5) is just equivalent to

$$
1-\rho_{1}=\left(1-\rho_{2}\right)^{\beta} \text {. }
$$

A homogeneous gamma process corresponds to a power-law shape function with $\beta=1$. Then the equivalent case just means that the two models share the same efficiency parameters $\left(\rho_{1}=\rho_{2}\right)$.

In case of a general power-law shape function, note that the "equivalent" case has a similar spirit to that detailed in (Doyen \& Gaudoin 2004, Property 4), where the authors match the minimal wear intensities of two imperfect repair models for recurrent events, based on the reduction of either virtual age or failure intensity.

The results for the equivalent case are summarized in the following proposition.
Proposition 3 Assume that $A(t)=\alpha t^{\beta}$ (with $\alpha, \beta>$ $0)$ and that $1-\rho_{1}=\left(1-\rho_{2}\right)^{\beta}$. Then:

1. $Z_{n T}^{(2)} \prec_{c v} Y_{n T}^{(1)}$ and $Y_{n T}^{(1)} \prec_{c x} Z_{n T}^{(2)}$ (which both entail that $\operatorname{var}\left(Z_{n T}^{(2)}\right) \geq \operatorname{var}\left(Y_{n T}^{(1)}\right)$ ) for all $n \geq 1$.

2. If $\beta \leq(\geq) 1$, then $Y_{t}^{(1)} \prec_{i c x}\left(\succ_{i c v}\right) Z_{t}^{(2)}$ (which entails that $\mathbb{E}\left(Y_{t}^{(1)}\right) \leq(\geq) \mathbb{E}\left(Z_{t}^{(2)}\right)$ ) for all $t \geq$ 0.

3. If $\beta \leq 1$, then $\operatorname{Var}\left(Z_{t}^{(2)}\right) \geq \operatorname{Var}\left(Y_{t}^{(1)}\right)$ for all $t \geq 0$.

\section{APPLICATION}

For illustration purpose of the previous results, the system is now supposed to provide some reward per unit time, which decreases when the deterioration level of the system increases. Based on classical functions used in the insurance literature ((Rolski, Schmidli, Schmidt, \& Teugels 1998)), we assume that the reward function is of the shape

$g(x)=\left(b_{1}-k_{1} e^{\alpha_{1} x}\right) \mathbf{1}_{\{0 \leq x \leq c\}}+\left(b_{2}-k_{2} e^{\alpha_{2} x}\right) \mathbf{1}_{\{c<x\}}$,

with $b_{1}, b_{2}, \alpha_{1}, \alpha_{2}, k_{1}, k_{2}, c>0$. The reward function is supposed to be continuous and positive on $[0, c)$, which implies that

$b_{2}-k_{2} e^{\alpha_{2} c}=b_{1}-k_{1} e^{\alpha_{1} c}>0$.

Also, we assume that $\alpha_{1} \leq \alpha_{2}$ and $k_{1} \leq k_{2}$ so that level $c$ appears as a critical level, from which the system becomes less performing.

With the previous assumptions, it is easy to check that $g$ is a concave function and that $g(x)>0$ if and only if $x<L=\frac{\ln \left(b_{2} / k_{2}\right)}{\alpha_{2}}$. Level $L$ hence appears as a critical threshold, from where the unitary reward becomes negative.

An example of reward function is plotted in Figure 5 with parameters $\alpha_{1}=0.1, b_{1}=11$ monetary units (m.u.), $\alpha_{2}=0.25, k_{1}=1$ (m.u.), $k_{2}=1$ (m.u.), $c=4$, and $b_{2}$ obtained through (7). With this dataset $L \simeq$ 10.01 .

The system is assumed to be preventively repaired each $T$ units of time according to an ARD1 or ARA1 model, with efficiency parameters $\rho_{1}$ and $\rho_{2}$, respectively. The accumulated reward on a time interval $[0, t]$ hence is

$R_{A R D 1}^{(1)}(t)=\mathbb{E}\left(\int_{0}^{t} g\left(Y_{s}^{(1)}\right) d s\right)=\int_{0}^{t} \mathbb{E}\left(g\left(Y_{s}^{(1)}\right)\right) d s$

with a similar expression for the accumulated reward $R_{A R A 1}^{(2)}(t)$ in the ARA1 case. 


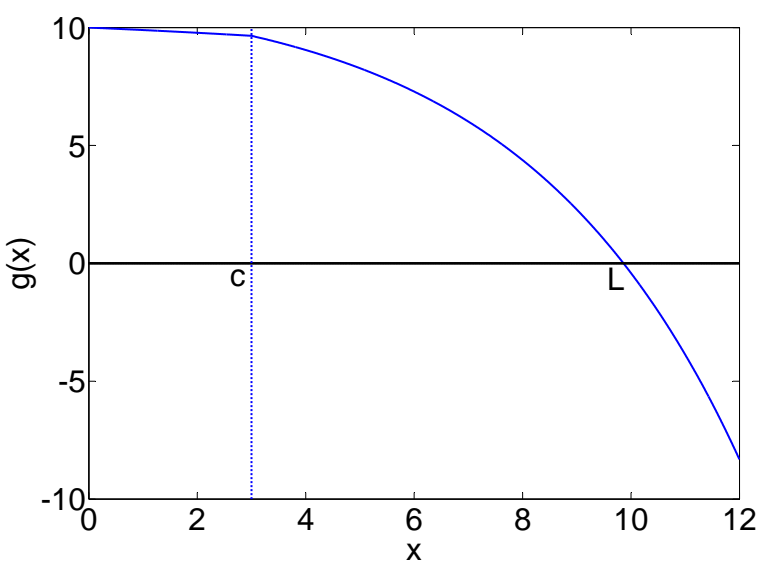

Figure 5: The reward function $g$ for $\alpha_{1}=0.1, b_{1}=11$ m.u., $\alpha_{2}=0.25, k_{1}=1$ m.u., $k_{2}=1$ m.u., $c=4$.

Considering for instance the equivalent case $\left(A(t)=\alpha t^{\beta}\right.$ and $\left.1-\rho_{1}=\left(1-\rho_{2}\right)^{\beta}\right)$ with $\beta \leq 1$, we easily derive from point 2 in Proposition 3 that

$\mathbb{E}\left(g\left(Y_{s}^{(1)}\right)\right) \geq \mathbb{E}\left(g\left(Z_{s}^{(2)}\right)\right)$

for all $s \in[0, t]$, using the fact that $-g$ is an increasing convex function. This immediately entails that

$R_{A R D 1}^{(1)}(t) \geq R_{A R A 1}^{(2)}(t)$ for all $t \geq 0$.

As a consequence, even if the "equivalent" case provides similar expected levels at maintenance times, they do not provide the same accumulated rewards on some given time interval. They may hence lead to different decisions in an applied context, for instance for optimizing maintenance strategies.

To better illustrate this difference, we now consider a preventive maintenance strategy, which we next optimize. The assumptions are the following:

- The unitary reward of the system per unit time is given by the reward function $g$ provided in (6). We recall that $L=\frac{\ln \left(b_{2} / k_{2}\right)}{\alpha_{2}}$ stands for a "critical" level, from where the reward becomes negative.

- The system is preventively repaired each $T$ units of time according to an ARD1 or ARA1 model (respective efficiency parameters: $\rho_{1}$ and $\rho_{2}$ ), up to the first maintenance time $K T$ where the deterioration level is observed to be beyond a given preventive threshold $M$ (with $M<L$ ). In that case, instead of an imperfect repair (ARA1 or ARD1), a replacement is performed at time $K T$ with cost $C_{c}$ m.u. when the level is beyond $L$ (corrective replacement) and $C_{p}$ m.u. when it is between $M$ and $L$ (preventive replacement).

The successive (corrective or preventive) replacements of the system appear as the points of a renewal process, and the long time (operating) profit rate per unit time is given by

$$
\begin{aligned}
& C_{A R D}(T, M) \\
& =\frac{1}{\mathbb{E}(K) T}\left[\mathbb{E}\left(\int_{0}^{K T} g\left(Y_{s}^{(1)}\right) d s\right)-C_{r}(\mathbb{E}(K)-1)\right. \\
& \left.\quad-C_{p} \mathbb{P}\left(M \leq Y_{K T}^{(1)}<L\right)-C_{c} \mathbb{P}\left(L \leq Y_{K T}^{(1)}\right)\right]
\end{aligned}
$$

for the ARD1 model with a similar expression for the ARA1 model $\left(C_{A R A}(T, M)\right)$. Due to the complexity of the model, there is not hope here to find conditions that ensure the dominance of one of the two functions $C_{A R D}(T, M)$ or $C_{A R A}(T, M)$ on the other. The comparison is hence made on a numerical example.

The degradation is modeled by a homogeneous gamma process with parameters $A(t)=1.3 t$ and $b=$ 0.8 . The parameters of the reward function $g$ are $\alpha_{1}=0.4, \alpha_{2}=0.5, b_{1}=800$ m.u., $k_{1}=1.05$ m.u., $k_{2}=1.07$ m.u., $c=8$, which implies $b_{2} \simeq 832.66$ m.u. and $L \simeq 13.31$. The repair efficiencies of the ARD1/ARA1 repairs are $\rho_{1}=\rho_{2}=0.9$, which corresponds to an equivalent case (homogeneous gamma process and $\rho_{1}=\rho_{2}$ ). Their common cost is $C_{r}=200$ m.u.. The cost of a preventive replacement is $C_{p}=$ 1000 m.u. whereas it is $C_{c}=1300$ m.u. for a corrective one. Figures $6 \mathrm{a}$ and $6 \mathrm{~b}$ show the profit rates for the maintained system under ARD1 and ARA1 repairs, respectively. These figures have been computed considering a grid of 10 points for $T$ from 1.14 to 4 and a grid of 13 points for $M$ from 1 to $L$ and 10000 simulations for each pair of points. For both ARD1 and ARA1 cases, the optimal maintenance strategy corresponds to $T^{o p t} \simeq 3.05$. However, the corresponding optimal maintenance levels are $M_{A R D}^{o p t} \simeq 9.21$ and $M_{A R A}^{o p t} \simeq 10.24$, and the optimal unitary rewards $C_{A R D}^{o p t} \simeq 673.94$ and $C_{A R A}^{o p t} \simeq 684.34$ m.u. per unit time, respectively. There hence is a difference of about $10 \%$ between the two optimal preventive levels, which may entail inappropriate decision in an applicative context, when considering one imperfect repair model or the other, whereas the effective behavior of the maintained system is closer to the other one.

\section{CONCLUSIONS AND FURTHER EXTENSIONS}

Two imperfect repair models for a degrading system have been proposed and compared in this paper. One is based on the reduction of the single deterioration level at maintenance times (ARD1), the other one on the reduction of both deterioration level and age (ARA1). They hence correspond to different maintenance actions, in an applicative context. The comparison of both location and spread have been made in terms of moments and stochastic ordering of the 


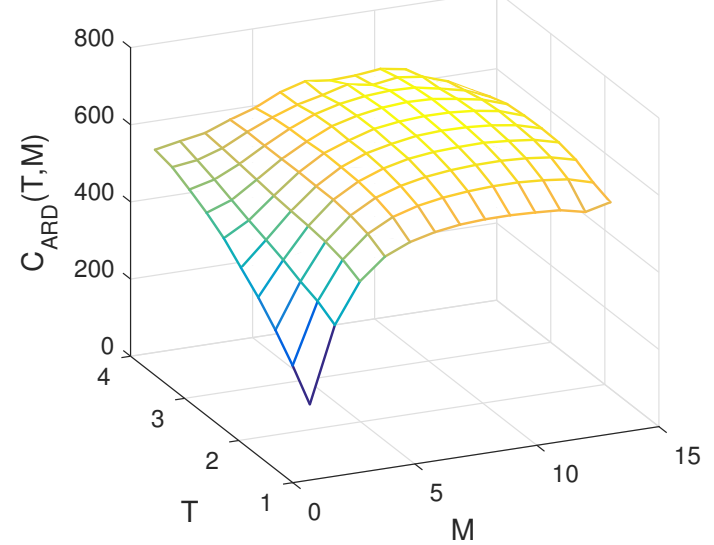

(a) ARD1 model

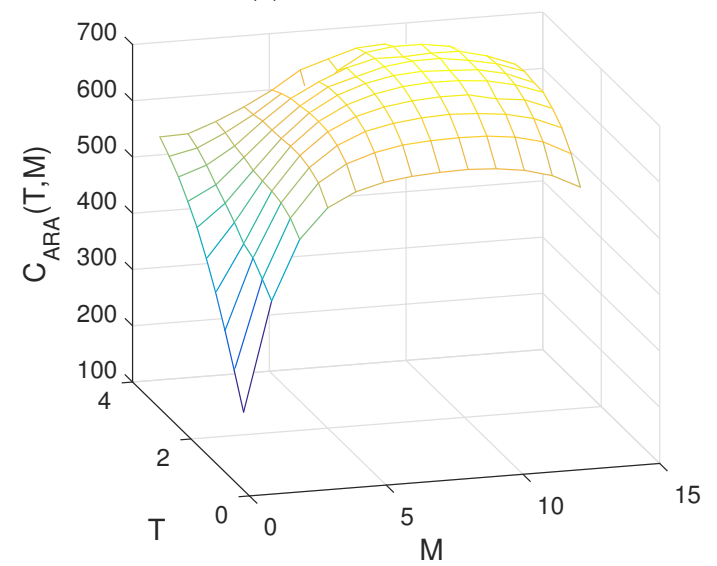

(b) ARA1 model

Figure 6: Operational profit rates, $\rho_{1}=\rho_{2}=0.9$

two resulting processes. It has been seen that the concavity/convexity of the shape function of the underlying gamma wear process plays a central role in the comparison results. This corresponds to intuition as a convex shape function (for instance) induces some increasingness property in the rate of deterioration over time. Hence, rejuvenating the system as in an ARA1 one action will decrease the rate of deterioration together with the deterioration level.

As for the stochastic comparison results, the paper focuses on the likelihood ratio order, which is well-known in reliability theory, but also on the (increasing/decreasing) convex/concave orders, which come from the insurance literature and seem a little less common in papers devoted to reliability theory. Clearly, other stochastic orders might be considered such as Laplace transform order or Excess Wealth order for instance. Other questions of interest concern the comparison of remaining lifetimes, considering the system as failed (or too degraded) when its deterioration level is beyond a fixed failure (critical) threshold. From a theoretical point of view, this seems a difficult issue in a general setting. One could then look at partial results on specific models.

Furthermore, the paper made an attempt for comparing the two types of imperfect repair including them in a global maintenance policy. Clearly, this subject requires further investigation for a better understanding of the practical consequences on the optimal policy of choosing one model of imperfect repair or the other, and typically, this would require a numerical study at a larger scale. Of course, it would also be of interest to consider other types of global maintenance policy (among the numerous ones developed in the literature, e.g. see van Noortwijk 2009), including one model of imperfect repair or the other.

Finally, a gamma deterioration model has been assumed in this paper. The stochastic comparison results between the two imperfect repair models deeply rely on the comparison properties of the gamma distribution, as summed up in Lemma 1 . As noted by the referee, a question of interest would be to revisit the comparison between the two imperfect repair models considering other deterioration models (such as Wiener processes with trend or other subordinators).

\section{REFERENCES}

Alaswad, S. \& Y. Xiang (2017). A review on condition-based maintenance optimization models for stochastically deteriorating system. Reliability Engineering \& System Safety 157, 54-63.

Caballé, N. C., I. T. Castro, C. J. Pérez, \& J. M. LanzaGutiérrez (2015). A condition-based maintenance of a dependent degradation-threshold-shock model in a system with multiple degradation processes. Reliability Engineering \& System Safety 134, 98-109.

Castro, I. T. \& S. Mercier (2016). Performance measures for a deteriorating system subject to imperfect maintenance and delayed repairs. Proceedings of the Institution of Mechanical Engineers, Part O: Journal of Risk and Reliability, 1-22.

Doyen, L. \& O. Gaudoin (2004). Classes of imperfect repair models based on reduction of failure intensity or virtual age. Reliability Engineering \& System Safety 84(1), 45-56.

Hong, H. P., W. Zhou, S. Zhang, \& W. Ye (2014). Optimal condition-based maintenance decisions for systems with dependent stochastic degradation of components. Reliability Engineering \& System Safety 121, 276-288.

Huynh, K. T., I. T. Castro, A. Barros, \& C. Bérenguer (2014). On the use of mean residual life as a condition index for condition-based maintenance decision-making. IEEE Transactions on Systems, Man, and Cybernetics: Systems 44(7), 877-893.

Kijima, M. (1989). Some results for repairable systems with general repair. Journal of Applied Probability 26(1), 89-102.

Mercier, S. \& I. T. Castro (2013). On the modelling of imperfect repairs for a continuously monitored gamma wear process through age reduction. Journal of Applied Probability 50(4), 1057-1076.

Mercier, S. \& I. T. Castro (submitted). Stochastic comparisons of imperfect maintenance models for a gamma deteriorating system.

Müller, A. \& D. Stoyan (2002). Comparison methods for stochastic models and risks. John Wiley \& Sons.

Rolski, J., H. Schmidli, V. Schmidt, \& J. Teugels (1998). Stochastic Processes for Insurance and Finance. John Wiley \& Sons.

Shaked, M. \& J. G. Shanthikumar (2007). Stochastic Orders. Springer.

van Noortwijk, J. (2009). A survey of the application of gamma processes in maintenance. Reliability Engineering \& System Safety 94(1), 2-21.

Zhang, M., G. Olivier, \& X. Min (2015). Degradation-based maintenance decision using stochastic filtering for systems under imperfect maintenance. European Journal of Operational Research 245, 531-541. 